\title{
.
}

\section{Growth and development}

\author{
With a year of publications under its belt, Nature Communications has established itself as an \\ accommodating venue for the natural sciences.
}

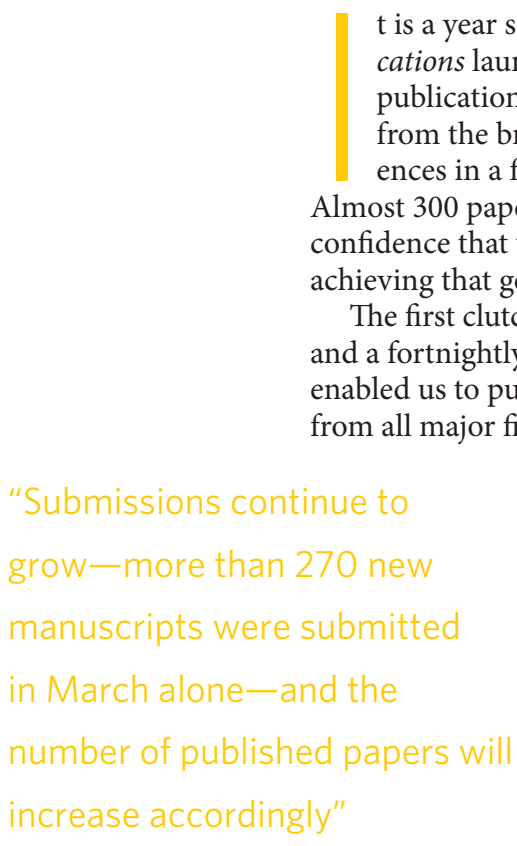

$\mathrm{t}$ is a year since Nature Communications launched, promising rapid publication of high-quality research from the breadth of the natural sciAlmost 300 papers later, we can say with me are well on the way to hieving that goal.

first clutch of Articles totalled 11 fortnightly publication schedule enabled us to publish a total of 150 papers, from all major fields, by the end of 2010 . But demand grew quickly, and, thanks to our accommodating publication model, we were able to make a rapid switch to a weekly schedule. Furthermore, our archive doubled in size during the first four months of 2011, and daily publication - which will further reduce

publication times-is likely to be introduced within the year.

Being a 'hybrid' publication, our flexibility also extends to the license under which work is published, and we are delighted that more than $45 \%$ of our content is freely available to all through one of two Creative Commons licenses. Naturally, we recognize that not all researchers will have access to the resources required for open-access publication, which is why authors can choose to publish their work without charge behind a subscription pay wall. We are sometimes asked whether the choice of publication license influences the decisions of the editors, and the answer is resolutely 'no'-editors are blind to the author's choice until the work is ready for acceptance.

And what of quality? Only time will tell, but no doubt our authors, and those whose papers we have regretfully rejected, will attest to the rigour of peer review at Nature Communications. Our selection criteria, upheld by our Editorial Advisory Panel, together with decisions made after peer review result in publication of approximately $20 \%$ of submissions-a number that suggests our aspirations of being a high-quality science journal with somewhat specialist appeal were well-grounded.

Nature Communications cannot rest on its laurels, however. Submissions continue to grow-more than 270 new manuscripts were submitted in March alone-and the number of published papers will increase accordingly. Our web technologies must therefore keep pace with this rapidly expanding archive. Indeed, since Nature Communications launched we have made our content available through mobile device applications to enable browsing on the go; launched a personalization tool on our homepage for users to customize their experience; and introduced an online commenting facility to encourage scientific dialogue.

So what does the future hold for Nature Communications? We are interested in understanding what you, the scientific community, would like to see (or indeed hear), and we encourage you to use the commenting tool at the foot of the HTML version of this article to pass on your thoughts. 